\title{
Polymorphisms of GSTP1, ERCC2 and TS-3'UTR are associated with the clinical outcome of mFOLFOX6 in colorectal cancer patients
}

\author{
KENSUKE KUMAMOTO, KEIICHIRO ISHIBASHI, NORIMICHI OKADA, YUSUKE TAJIMA, \\ KOUKI KUWABARA, YOICHI KUMAGAI, HIROYUKI BABA, NORIHIRO HAGA and HIDEYUKI ISHIDA \\ Department of Digestive Tract and General Surgery, Saitama Medical Center, \\ Saitama Medical University, Kawagoe, Saitama 350-8550, Japan
}

Received December 30, 2012; Accepted June 5, 2013

DOI: $10.3892 /$ ol.2013.1467

\begin{abstract}
The aim of the current study was to examine whether polymorphisms in drug metabolism genes have any clinical impact on patients treated with 5-fluorouracil (FU)/oxaliplatin for metastatic colorectal cancer (MCRC). In total, 63 patients with MCRC were recruited and treated with a modified FOLFOX6 (mFOLFOX6) treatment as a first-line chemotherapy. Polymorphisms in five drug metabolism genes and two DNA-repair genes were assessed in these patients using polymerase chain reaction (PCR), a PCR restriction fragment length polymorphism (PCR-RFLP) technique or invader techniques. These included a 28-bp tandem repeat in the 5'-untranslated region (UTR) and 6-bp deletions in the 3'-UTR of thymidylate synthase (TS), methylenetetrahydrofolate reductase (MTHFR; Ala677Val), glutathione S-transferase $\pi$ (GSTP1; IIe105Val), GST $\theta 1$ (GSTT1; deletion) and GST $\mu 1$ (GSTM1; deletion) and the two DNA-repair genes, excision repair cross-complementing-1 (ERCC1; Asp118Asn) and ERCC2 (Lys751Gln). The correlation between these polymorphisms and the clinical outcome, including drug response, progression-free survival (PFS), overall survival (OS) and the incidence of peripheral neuropathy, were evaluated. Patients with the GSTP1-105 A/A genotype had poor responses to mFOLFOX6 treatment compared with those with the GSTP1-105 A/G and G/G genotypes $(\mathrm{P}=0.01)$. The median PFS of patients with the ERCC2-751 A/A genotype tended to be longer than that of patients with the ERCC2-751 A/C genotype $(\mathrm{P}=0.05)$. Patients with the TS-3'-UTR -6/-6 genotype had a significantly longer OS compared with patients with other genotypes $(\mathrm{P}=0.003)$. A statistically significant association between the incidence of
\end{abstract}

Correspondence to: Dr Kensuke Kumamoto, Department of Digestive Tract and General Surgery, Saitama Medical Center, Saitama Medical University, 1981 Kamoda, Kawagoe, Saitama 350-8550, Japan

E-mail:kumamotk@saitama-med.ac.jp

Key words: FOLFOX, colorectal cancer, polymorphism peripheral neuropathy higher than grade 2 and the GSTP1-105 $(\mathrm{P}=0.03)$ and $G S T M 1$ genotypes $(\mathrm{P}=0.02)$ was identified by multivariate logistic regression analyses. Results demonstrated that polymorphisms in GSTP1-105, ERCC2-751 and the 3'-UTR of TS may be a statistically significant predictors of clinical outcome. GSTP1-105 and GSTM1 genotypes may be useful markers of severe peripheral neuropathy in MCRC patients treated with 5-FU/oxaliplatin as first-line chemotherapy.

\section{Introduction}

Advances in chemotherapeutic regimens for metastatic colorectal cancer (MCRC) patients, including FOLFOX treatment comprising of a combination of 5-fluorouracil (FU)/leucovorin (LV) and oxaliplatin, have improved overall survival (OS) $(1,2)$. It has been reported that the efficacy rate of FOLFOX treatment varies between 20 and $50 \%$ in MCRC patients (1,3-5). Individuals who receive chemotherapy commonly suffer from side effects, including myelosuppression, nausea, diarrhea and peripheral neuropathy (1-3). Numerous patients undergoing FOLFOX treatment have complained of oxaliplatin-induced peripheral neuropathy. Therefore, several markers for predicting the efficacy of FOLFOX treatment have been investigated to identify patients with favorable treatment prognoses. Gene expression analysis, associated with the metabolism of 5-FU and oxaliplatin, has been intensively studied (6-9). It has been reported that thymidylate synthase (TS) and thymidine phosphorylase $(T P)$ mRNA expression levels are useful markers for predicting the efficacy of FOLFOX treatment in CRC patients with liver metastasis (10). In addition, advances in molecular biology indicate that a number of drug metabolism genes have polymorphisms that alter levels of expression. Among these, polymorphisms have been identified in $T S$, excision repair cross-complementing-1 (ERCC1) and ERCC2, glutathione S-transferase $\pi$ (GSTP1), GST $\theta 1$ (GSTT1), GST $\mu 1$ (GSTM1) and methylenetetrahydrofolate reductase (MTHFR), which exert functions in drug metabolism and antidotal effects on the 5-FU and oxaliplatin pathways. Studies have demonstrated that specific polymorphisms of these genes are associated with the efficacy of FOLFOX treatment in MCRC patients (11-14). 
TS and MTHFR are associated with the metabolism of 5-FU, indicating that their altered expression affects the response to 5-FU-based chemotherapy. The enzyme product of TS is critical for catalyzing the methylation of deoxyuridine-5'-monophosphate to deoxythymidine-5'-monophosphate in de novo DNA synthesis. Fluorodeoxyuridine monophosphate (FdUMP), the metabolic product of 5-FU, forms complexes with TS and 5,10-methylenetetrahydrofolate, resulting in the inhibition of DNA synthesis. Two polymorphisms have been identified in $T S$, a variable length tandem repeat polymorphism in the 5'-untranslated region (UTR) that consists of two or three 28-bp repeated sequences and a 6-bp insertion/deletion (6+/6-) in the 3'-UTR. A number of studies have described correlations between genotype patterns of polymorphisms in TS and the efficacy of FOLFOX treatment for MCRC patients (11-14). However, current evidence is insufficient to confirm a statistically significant correlation. MTHFR is important for folate metabolism and catalyzing the conversion of 5,10-methylenetetrahydrofolate to 5-methyltetrahydrofolate. Two important polymorphisms in MTHFR, C677T and A1298C, have been studied (12-14) and have been identified to affect the enzyme activity of MTHFR $(15,16)$, leading to the accumulation of 5,10-methylenetetrahydrofolate and the enhanced sensitivity of 5-FU by forming complexes with TS and FdUMP. Studies have described the correlation among these polymorphisms and the efficacy of FOLFOX treatment for MCRC patients $(12,14)$.

The expression levels of ERCC1, ERCC2, GSTP1, GSTT1 and GSTM1 have been hypothesized to be associated with the efficacy of platinum compounds, including cisplatin and oxaliplatin. ERCC-1 and -2 are involved in DNA repair and tolerance of DNA damage through the nucleotide excision repair pathway. The enhanced expression of these proteins may lead to the resistance to platinum drugs. A common $\mathrm{C}$ to $\mathrm{T}$ transition at codon 118 of ERCC1 has been shown to increase expression in patients with the $\mathrm{T} / \mathrm{T}$ genotype compared with patients with the $\mathrm{C} / \mathrm{T}$ or $\mathrm{C} / \mathrm{C}$ genotypes (17), despite the $\mathrm{T} / \mathrm{T}$ polymorphism producing the identical amino acid, asparagine. Studies have revealed that patients with the T/T genotype have a poor outcome compared with patients with the $\mathrm{C} / \mathrm{T}$ or $\mathrm{C} / \mathrm{C}$ genotypes (13). During oxaliplatin-based chemotherapy, the prognosis for MCRC patients with the $\mathrm{C} / \mathrm{C}$ genotype is more encouraging than that of patients with other genotypes $(11,18,19)$. The xeroderma pigmentosum group D (XPD) gene, ERCC2, has three common polymorphisms in codons 156, 312 and 751. The polymorphism at codon 751 (A $>C$ : Lys $>$ Gln) is associated with the clinical outcome of MCRC patients receiving FOLFOX treatment $(13,20)$.

The GST family includes at least five subclasses with major biological roles in the detoxification of genotoxic compounds. GSTP1, GSTT1 and GSTM1 genotypes have been extensively studied for drug response, including oxaliplatin-based treatment (21-23). A single nucleotide polymorphism at codon 105 (A $>$ G: Ile $>$ Val) of GSTPl affects enzyme activity (24). Several studies have demonstrated that among MCRC patients receiving oxaliplatin-based treatment, patients with GSTP1-105 A/G and G/G genotypes have a more favorable outcome compared with patients with the GSTP1-105 A/A genotype (21-23).

In the present study, correlations were identified between the polymorphism patterns of TS, MTHFR, ERCC1, ERCC2, GSTP1, GSTT1 and GSTM1 and the clinical outcome,
Table I. Patient characteristics.

\begin{tabular}{|c|c|}
\hline Parameter & Value \\
\hline Total patients, $\mathrm{n}$ & 63 \\
\hline Gender, males:females & $41: 22$ \\
\hline Age, years ${ }^{\mathrm{a}}$ & $65(32-84)$ \\
\hline \multicolumn{2}{|l|}{ Location, $\mathrm{n}$} \\
\hline Colon/rectosigmoid & 43 \\
\hline Rectum & 20 \\
\hline \multicolumn{2}{|l|}{ Performance status, $n$} \\
\hline 0 & 39 \\
\hline 1 & 21 \\
\hline 2 & 3 \\
\hline \multicolumn{2}{|l|}{ Number of target organ(s) } \\
\hline 1 & 26 \\
\hline$>2$ & 37 \\
\hline \multicolumn{2}{|l|}{ Target organ, $\mathrm{n}$} \\
\hline Liver & 43 \\
\hline Lung & 18 \\
\hline Lymph node & 16 \\
\hline Peritoneum & 13 \\
\hline Others & 7 \\
\hline Cycles of mFOLFOX6 therapy, $\mathrm{n}^{\mathrm{a}}$ & $10.0(4-39)$ \\
\hline Relative dose intensity, $\%^{a}$ & $75.0(28.1-100)$ \\
\hline \multicolumn{2}{|l|}{ Response, $\mathrm{n}$} \\
\hline CR & 3 \\
\hline PR & 23 \\
\hline $\mathrm{SD}$ & 24 \\
\hline PD & 13 \\
\hline \multicolumn{2}{|l|}{ Second line chemotherapy, $n$} \\
\hline FOLFIRI & 29 \\
\hline FOLFIRI + bevacizumab & 15 \\
\hline Other & 3 \\
\hline Best supportive care & 16 \\
\hline
\end{tabular}

${ }^{a}$ Median (range). CR, complete response; PR, partial response; $\mathrm{SD}$, stable disease; $\mathrm{PD}$, progressive disease; FOLFIRI, folinic acid/5-FU/irinotecan.

including the incidence of peripheral neuropathy, in Japanese MCRC patients who were treated with modified FOLFOX6 (mFOLFOX6).

\section{Materials and methods}

Patients and clinical procedures. The current study was performed in accordance with the ethical guidelines for clinical studies with approval from the institutional ethics committee. Informed consent was obtained from all individuals.

The subjects included $63 \mathrm{CRC}$ patients (22 females and 41 males) who received mFOLFOX6 treatment as first-line chemotherapy between 2005 and 2009. The mFOLFOX6 regimen was comprised of intravenous infusions of oxaliplatin 
Table II. Characteristics of polymorphisms with primer sequences and restriction enzymes.

\begin{tabular}{|c|c|c|c|c|c|}
\hline Site & Polymorphism & Genotype & $\begin{array}{l}\text { Restriction } \\
\text { enzymes }\end{array}$ & Primers & $\begin{array}{l}\text { Detection } \\
\text { method }\end{array}$ \\
\hline TS 5'-UTR & VNTR & $2 \mathrm{R}$ or $3 \mathrm{R}$ alleles & & $\begin{array}{l}\text { 5'-AGGCGCGCGGAAGGGGTCCT-3' } \\
\text { 5'-TCCGAGCCGGCCACAGGCAT-3' }\end{array}$ & PCR \\
\hline TS 3'-UTR & $\begin{array}{c}6 \text { bp insertion/ } \\
\text { deletion }\end{array}$ & $6+/ 6-$ & Dral & $\begin{array}{l}\text { 5'-CAAATCTGAGGGAGCTGAGT-3' } \\
\text { 5'-CAGATAAGTGGCAGTACAGA-3' }\end{array}$ & PCR-RFLP \\
\hline MTHFR (exon 4) & SNP & C/T, Ala677Val & $\operatorname{Hinfi}$ & $\begin{array}{l}\text { 5'-TGAAGGAGATGTCTGCGGGA-3' } \\
\text { 5'-AGGACGGTGCGGTGAGAGTG-3' }\end{array}$ & $\begin{array}{l}\text { Invader } \\
\text { method }\end{array}$ \\
\hline$E R C C 1$ (exon 4) & SNP & $\mathrm{C} / \mathrm{T}, \mathrm{Asn} 118 \mathrm{Asn}$ & Maell & $\begin{array}{l}\text { 5'-GAGAGGGCTGAGCTGGAGACAG-3' } \\
\text { 5'-CCAGCACATAGTCGGGAATTACGTC-3' }\end{array}$ & PCR-RFLP \\
\hline$E R C C 2$ (exon 23) & SNP & A/C, Lys751Gln & Mboll & $\begin{array}{l}\text { 5'-CAGGTGAGGGGGACATCTG-3' } \\
\text { 5'-CTCTCCCTTTCCTCTGTTC-3' }\end{array}$ & PCR-RFLP \\
\hline GSTP1 (exon 5) & SNP & A/G, IIe105Val & Mspl & $\begin{array}{l}\text { 5'-ACCCCAGGGCTCTATGGGAA-3' } \\
\text { 5'-TGAGGGCACAAGCCCCT-3' }\end{array}$ & PCR-RFLP \\
\hline GSTT1 & Deletion & $\pm^{\mathrm{a}}$ & & $\begin{array}{l}\text { 5'-TTCCTTACTGGTCCTCCTCACATCTC-3' } \\
\text { 5'-TCACCGGATCATGGCCAGCA-3' }\end{array}$ & PCR \\
\hline GSTM1 & Deletion & $\pm^{\mathrm{a}}$ & & $\begin{array}{l}\text { 5'-GAACTCCCTGAAAAGCTAAAGC-3' } \\
\text { 5'-GTTGGGCTCAAATATACGGTGG-3' }\end{array}$ & PCR \\
\hline
\end{tabular}

${ }^{\mathrm{a}}$ Genotype was defined as positive if at least one copy of the gene was present. TS, thymidylate synthase; VNTR, variable number tandem repeat; 2R/3R, two/three 28-bp repeated sequences; UTR, untranslated region; MTHFR, methylenetetrahydrofolate reductase; ERCC, excision repair cross-complementing; SNP, single uncleotide polymorphism; GSTP1, glutathione S-transferase $\pi$; GSTT1, glutathione S-transferase $\theta 1$; GSTM1, glutathione S-transferase $\mu 1$; PCR, polymerase chain reaction; RFLP, restriction fragment length polymorphism.

$\left(85 \mathrm{mg} / \mathrm{m}^{2}\right)$ and $\mathrm{LV}\left(200 \mathrm{mg} / \mathrm{m}^{2}\right)$ for $2 \mathrm{~h}$, followed by a rapid intravenous bolus infusion of 5 -FU $\left(400 \mathrm{mg} / \mathrm{m}^{2}\right)$ for $5 \mathrm{~min}$ and a continuous intravenous infusion of 5 -FU $\left(2,400 \mathrm{mg} / \mathrm{m}^{2}\right)$ for $46 \mathrm{~h}$. This regimen was repeated every 2 weeks. Table I presents the patient characteristics. The median age of the patients was 65 years old (range, 32-84 years old). The primary site was the colon/rectosigmoid in 43 patients and the rectum in 20 patients. Performance status (PS), determined according to the method of the Eastern Cooperative Oncology Group was 0 in 39 patients, 1 in 21 patients and 2 in 3 patients. The target lesions were located in the liver of 43 patients, the lungs of 18 patients, the peritoneum of 13 patients and the lymph nodes of 16 patients, while in 7 patients the target lesions were detected in other locations. The median number of oxaliplatin doses was 10 (range, 4-39) and the median relative dose intensity of oxaliplatin was $75 \%$ (range, 28.1-100\%). The response to mFOLFOX6 treatment was evaluated during 4-6 courses of treatment according to the Response Evaluation Criteria in Solid Tumors (version 1.1) (25). Complete response was observed in 3 patients, partial response in 23 patients, stable disease in 24 patients and progressive disease in 13 patients. Adverse events were graded according to the Common Terminology Criteria for Adverse Events (version 3.0). When an adverse event of >grade 3 severity occurred, mFOLFOX6 therapy was suspended until the severity of the reaction improved to $<$ grade 2. When mFOLFOX6 therapy was resumed, doses of oxaliplatin were reduced to $70-80 \%$ of the previous dose.

Of the 63 patients with CRC, 44 received folinic acid/5-FU/irinotecan (FOLFIRI) either with $(n=15)$ or without bevacizumab $(\mathrm{n}=29)$ as a second-line chemotherapy treatment. The FOLFIRI regimen comprised of intravenous infusions of irinotecan $\left(150 \mathrm{mg} / \mathrm{m}^{2}\right)$ and $\mathrm{LV}\left(200 \mathrm{mg} / \mathrm{m}^{2}\right)$ for $2 \mathrm{~h}$, followed by a rapid intravenous bolus infusion of $5-\mathrm{FU}\left(400 \mathrm{mg} / \mathrm{m}^{2}\right)$ for $5 \mathrm{~min}$ and a continuous intravenous infusion of 5-FU $\left(2,400 \mathrm{mg} / \mathrm{m}^{2}\right)$ for $46 \mathrm{~h}$, administered every 2 weeks. A total of 16 patients were observed without administration of additional treatment.

DNA extraction and analysis of polymorphisms. Genomic DNA was extracted from 23 blood samples and 40 normal colonic mucosae from each enrolled patient using the QIAamp DNA Blood and QIAamp DNA Mini kits (Qiagen, Tokyo, Japan). Polymorphisms were analyzed by polymerase chain reaction (PCR), a PCR restriction fragment length polymorphism (PCR-RFLP) technique and a PCR-invader method. Primer sequences and restriction enzymes of all genes examined are presented in Table II.

Statistical analysis. Continuous data are presented as the median and range. Mann-Whitney U, Fisher's exact probability and $\chi^{2}$ tests were used where applicable. A survival analysis was conducted using the Kaplan-Meier method. The log-rank test was used to determine the significance of the survival curves. The OS period was calculated between the time of surgery and the date of mortality of any cause. OS was censored from the time of the individuals last visit to the hospital or December 2010, depending on which was the first event. Logistic regression was used to determine independent predictors of adverse events. $\mathrm{P}<0.05$ was considered to indicate a statistically significant difference. All statistical analyses were performed using a statistical software package (StatFlex ver.6.0; Artech, Osaka, Japan). 
Table III. Frequency of polymorphisms, response rate and median PFS and OS.

\begin{tabular}{|c|c|c|c|c|c|c|c|}
\hline Gene & $\begin{array}{c}\text { Patients } \mathrm{n}=63, \\
\mathrm{n}(\%)\end{array}$ & $\begin{array}{c}\text { Responder, } \\
\text { n }(\%)\end{array}$ & P-value & $\begin{array}{l}\text { Median PFS } \\
\text { months }\end{array}$ & P-value & $\begin{array}{l}\text { Median } \mathrm{OS}^{\mathrm{b}}, \\
\text { months }\end{array}$ & P-value \\
\hline TS-5'UTR & & & 0.11 & & 0.56 & & 0.650 \\
\hline $3 R / 3 R$ & $46(73)$ & $18(39)$ & & 8.6 & & 27.0 & \\
\hline $2 \mathrm{R} / 3 \mathrm{R}$ & $13(21)$ & $8(62)$ & & 9.9 & & 25.4 & \\
\hline $2 \mathrm{R} / 2 \mathrm{R}$ & $3(5)$ & $0(0)$ & & 11.1 & & 31.8 & \\
\hline Unknown & $1(2)$ & & & & & & \\
\hline TS-3'UTR & & & 0.93 & & 0.48 & & 0.003 \\
\hline$-6 /-6$ & $24(38)$ & $10(42)$ & & 11.6 & & 34.4 & \\
\hline$-6 /+6$ & $29(46)$ & $13(45)$ & & 8.3 & & 24.4 & \\
\hline$+6 /+6$ & $10(16)$ & $3(30)$ & & 10.7 & & 14.8 & \\
\hline MTHFR-677 & & & 0.70 & & 0.80 & & 0.860 \\
\hline $\mathrm{C} / \mathrm{C}$ & $26(41)$ & $12(46)$ & & 9.9 & & 27.4 & \\
\hline $\mathrm{C} / \mathrm{T}$ & $30(48)$ & $11(37)$ & & 8.1 & & 27.0 & \\
\hline $\mathrm{T} / \mathrm{T}$ & $6(10)$ & $3(50)$ & & 8.3 & & 24.4 & \\
\hline Unknown & $1(2)$ & & & & & & \\
\hline ERCC1-118 & & & 0.71 & & 0.63 & & 0.380 \\
\hline $\mathrm{C} / \mathrm{C}$ & $30(48)$ & $11(37)$ & & 9.9 & & 27.4 & \\
\hline $\mathrm{C} / \mathrm{T}$ & $23(37)$ & $11(48)$ & & 8.1 & & 22.5 & \\
\hline $\mathrm{T} / \mathrm{T}$ & $10(16)$ & $4(40)$ & & 8.3 & & 32.9 & \\
\hline ERCC2-751 & & & 0.95 & & 0.05 & & 0.690 \\
\hline $\mathrm{A} / \mathrm{A}$ & $58(92)$ & $24(41)$ & & 10.3 & & 25.5 & \\
\hline $\mathrm{A} / \mathrm{C}$ & $5(8)$ & $2(40)$ & & 6.1 & & 29.2 & \\
\hline $\mathrm{C} / \mathrm{C}$ & $0(0)$ & & & & & & \\
\hline GSTP1-105 & & & 0.05 & & 0.41 & & 0.260 \\
\hline $\mathrm{A} / \mathrm{A}$ & $44(70)$ & $14(32)$ & $0.01^{\mathrm{a}}$ & 8.6 & & 24.4 & \\
\hline $\mathrm{A} / \mathrm{G}$ & $16(25)$ & $11(69)$ & & 7.8 & & 31.1 & \\
\hline $\mathrm{G} / \mathrm{G}$ & $2(3)$ & $1(50)$ & & 11.8 & & 46.3 & \\
\hline Unknown & $1(2)$ & & & & & & \\
\hline GSTT1 & & & 0.83 & & 0.47 & & 0.840 \\
\hline Positive & $30(48)$ & $13(43)$ & & 8.1 & & 25.5 & \\
\hline Negative & $32(51)$ & $13(41)$ & & 10.3 & & 27.1 & \\
\hline Unknown & $1(2)$ & & & & & & \\
\hline GSTM1 & & & 0.73 & & 0.89 & & 0.480 \\
\hline Positive & $23(37)$ & $9(39)$ & & 7.4 & & 22.5 & \\
\hline Negative & $39(62)$ & $17(44)$ & & 10.7 & & 27.4 & \\
\hline Unknown & $1(2)$ & & & & & & \\
\hline
\end{tabular}

${ }^{\mathrm{a} C o m p a r i s o n}$ between A/A and A/G + G/G, ${ }^{\mathrm{K}}$ Kaplan-meier methods, logrank test. PFS, progression-free survival; OS, overall survival; TS, thymidylate synthase; VNTR, variable number tandem repeat; UTR, untranslated region; MTHFR, methylenetetrahydrofolate reductase; ERCC, excision repair cross-complementing; SNP, single uncleotide polymorphism; GSTP1, glutathione S-transferase $\pi$; GSTT1, glutathione S-transferase $\theta 1$; GSTM1, glutathione S-transferase $\mu 1$.

\section{Results}

Correlation between polymorphisms in TS, MTHFR, ERCC1, ERCC2, GSTP1, GSTT1 and GSTM1 and the response rate to mFOLFOX6 treatment. Polymorphisms of GSTP1-105 were shown to significantly correlate with the efficacy of mFOLFOX6 treatment (Table III). The frequencies of GSTP1-105 A/A, A/G and G/G genotypes were 70, 25 and 3\%, respectively. In the responder group, fewer patients expressed the GSTP1-105 A/A genotype than the GSTP1-105 A/G or G/G genotypes $(\mathrm{P}=0.01)$. No significant differences were identified between the polymorphisms of other genes and the efficacy of mFOLFOX6 treatment.

Correlation between polymorphisms in TS, MTHFR, ERCC1, ERCC2, GSTP1, GSTT1 and GSTM1 and PFS and OS in $M C R C$ patients treated with mFOLFOX6. Frequencies of the ERCC2-751 A/A, A/C and C/C genotypes were 92, 8 and $0 \%$, 
Table IV. Correlation between peripheral neuropathy and polymorphisms.

\begin{tabular}{|c|c|c|c|c|c|c|c|c|}
\hline \multirow[b]{2}{*}{ Gene } & \multirow{2}{*}{$\begin{array}{c}\text { Patients, } \\
\text { n }\end{array}$} & \multirow{2}{*}{$\begin{array}{c}\text { Patients with } \\
>\text { Grade } 2, \mathrm{n}(\%)\end{array}$} & \multicolumn{3}{|c|}{ Univariate regression analysis } & \multicolumn{3}{|c|}{ Multivariate regression analysis } \\
\hline & & & OR & $95 \% \mathrm{CI}$ & P-value & OR & $95 \% \mathrm{CI}$ & P-value \\
\hline \multicolumn{9}{|l|}{ TS-5'UTR } \\
\hline $3 R / 3 R$ & 46 & $32(70)$ & 1 & & & & & \\
\hline $2 \mathrm{R} / 2 \mathrm{R}, 2 \mathrm{R} / 3 \mathrm{R}$ & 16 & $11(69)$ & 0.962 & $0.281-3.289$ & 0.95 & & & \\
\hline \multicolumn{9}{|l|}{ TS-3'UTR } \\
\hline$-6 /-6$ & 24 & $19(79)$ & 1 & & & & & \\
\hline$-6 /+6,+6 /+6$ & 39 & $25(64)$ & 0.470 & $0.144-1.533$ & 0.21 & & & \\
\hline \multicolumn{9}{|l|}{ MTHFR-677 } \\
\hline $\mathrm{C} / \mathrm{C}$ & 26 & $19(73)$ & 1 & & & & & \\
\hline $\mathrm{C} / \mathrm{T}, \mathrm{T} / \mathrm{T}$ & 36 & $24(67)$ & 0.737 & $0.243-2.235$ & 0.59 & & & \\
\hline \multicolumn{9}{|l|}{ ERCC1-118 } \\
\hline $\mathrm{C} / \mathrm{C}$ & 30 & $20(67)$ & 1 & & & & & \\
\hline $\mathrm{C} / \mathrm{T}, \mathrm{T} / \mathrm{T}$ & 33 & $24(73)$ & 1.333 & $0.453-3.921$ & 0.60 & & & \\
\hline \multicolumn{9}{|l|}{ ERCC2-751 } \\
\hline $\mathrm{A} / \mathrm{A}$ & 58 & $41(71)$ & 1 & & & & & \\
\hline $\mathrm{A} / \mathrm{C}, \mathrm{C} / \mathrm{C}$ & 5 & $3(60)$ & 0.622 & $0.095-4.062$ & 0.62 & & & \\
\hline \multicolumn{9}{|l|}{ GSTP1-105 } \\
\hline $\mathrm{A} / \mathrm{A}$ & 44 & $27(61)$ & 1 & & & 1 & & \\
\hline $\mathrm{A} / \mathrm{G}, \mathrm{G} / \mathrm{G}$ & 18 & $16(89)$ & 5.037 & $1.027-24.712$ & 0.05 & 6.084 & $1.150-32.175$ & 0.03 \\
\hline \multicolumn{9}{|l|}{ GSTT1 } \\
\hline Positive & 30 & $21(70)$ & 1 & & & & & \\
\hline Negative & 32 & $22(69)$ & 0.943 & $0.320-2.778$ & 0.92 & & & \\
\hline \multicolumn{9}{|l|}{ GSTMI } \\
\hline Positive & 23 & $12(52)$ & 1 & & & 1 & & \\
\hline Negative & 39 & $31(79)$ & 3.546 & $1.149-10.989$ & 0.03 & 4.202 & $1.253-14.085$ & 0.02 \\
\hline
\end{tabular}

TS, thymidylate synthase; UTR, untranslated region; MTHFR, methylenetetrahydrofolate reductase; ERCC, excision repair cross-complementing; GSTP1, glutathione S-transferase $\pi$; GSTT1, glutathione S-transferase $\theta 1$; GSTM1, glutathione S-transferase $\mu 1$; OR, odds ratio; $\mathrm{CL}$, confidence interval.

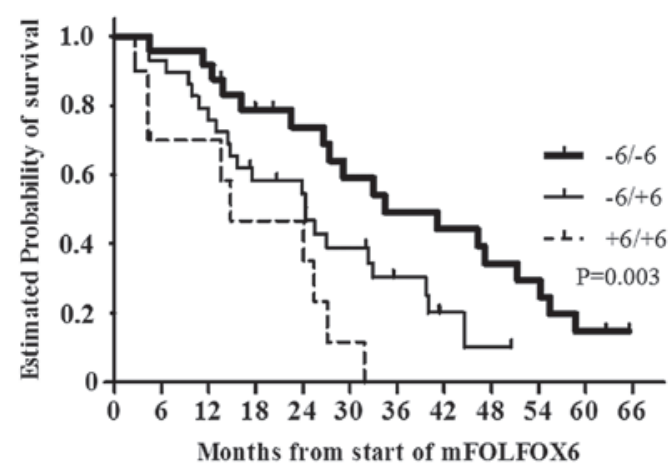

Figure 1. Overall survival (OS) in metastatic colorectal cancer patients with TS-3'-UTR $-6 /-6(n=24),-6 /+6(n=29)$ and $+6 /+6(n=10)$ genotypes. The median OS of patients with the TS-3'-UTR $-6 /-6,-6 /+6$ and $+6 /+6$ genotypes was 34.4, 24.4 and 14.8 months, respectively. TS, thymidylate synthase; UTR untranslated region.

respectively (Table III). The median PFS of patients with the ERCC2-751 A/A genotype was longer than that of patients with the ERCC2-751 A/C genotype (10.3 and 6.1 months, respectively; $\mathrm{P}=0.05)$. There was no correlation between polymorphisms of other genes and PFS. The median OS of the patients with the TS-3'-UTR -6/-6 (n=24), -6/+6 (n=29) and $+6 /+6(n=10)$ genotypes was $34.4,24.4$ and 14.8 months, respectively. The OS of the patients with the TS-3'-UTR -6/-6 genotype was significantly longer compared with that of the patients with other genotypes $(\mathrm{P}=0.003$; Fig. 1).

Correlation between polymorphisms in TS, MTHFR, ERCC1, ERCC2, GSTP1, GSTT1 and GSTM1 and incidence of peripheral neuropathy in patients treated with MFOLFOX6. The incidence of peripheral neuropathy of grades $2(n=42)$ and $3(n=2)$ was found to significantly correlate with the GSTP1-105 ( $\mathrm{P}=0.05)$ and GSTM1 $(\mathrm{P}=0.03)$ genotypes, as identified by univariate regression analyses (Table IV). Peripheral neuropathy occurred in the majority of patients with the GSTP1-105 A/G and G/G genotypes compared with patients with the GSTP1-105 A/A genotype. Individuals who were GSTM1-negative also had peripheral neuropathy, whereas individuals who were GSTM1-positive did not. A statistically significant correlation between the incidence of peripheral 
neuropathy higher than grade 2 and the GSTP1-105 ( $\mathrm{P}=0.03)$ and GSTM1 ( $\mathrm{P}=0.02)$ genotypes was determined using multivariate regression analysis.

\section{Discussion}

In the present study, specific polymorphisms of genes involved in 5-FU/oxaliplatin metabolism were demonstrated to be significantly associated with the clinical outcome of Japanese MCRC patients who received first-line chemotherapy with mFOLFOX6. The response to mFOLFOX6 treatment in patients with the GSTPI-105 A/G and G/G genotypes was significantly improved compared with that of patients with the GSTPI-105 A/A genotype. In addition, the ERCC2-751 and TS-3'-UTR genotypes were shown to significantly correlate with PFS and OS, respectively. The results indicated that polymorphisms in the oxaliplatin-associated genes, GSTP1-105 and ERCC2-751, were hypothesized to be important for the prediction of primary clinical outcome, including drug responses and PFS, for MCRC patients treated with mFOLFOX6. Second- and third-line chemotherapy regimens also affected OS. Of the 63 patients, 44 were treated with FOLFIRI following the FOLFOX regimen and 5-FU treatment continued throughout. Therefore, it is possible that polymorphisms in the genes involved in 5-FU metabolism contribute to OS in long-term observations.

Previous studies have revealed that GSTPI-105 genotypes are associated with the clinical outcome of MCRC patients who receive 5-FU/oxaliplatin as first-line chemotherapy (11-13,21-23). As GSTP1 expression is enhanced in CRC (26) it has been hypothesized to be involved in the resistance to platinum compounds (27). The enzyme activity of the GSTPI-105 A/G and G/G genotypes is lower than that of the GSTP1-105 A/A genotype (24). In addition, clinical assessments of the correlation between the GSTPI genotype and the clinical outcome in MCRC patients treated with 5-FU/oxaliplatin appears to be consistent with basic studies. In the present study, patients with the GSTPI-105 A/G and G/G genotypes were revealed to have a significantly improved response to mFOLFOX6 treatment when compared with patients with the GSTP1-105 A/A genotype. Previous studies have indicated that the GSTP1-105 A/G and $\mathrm{G} / \mathrm{G}$ genotypes are significantly more common than the GSTPI-105 A/A genotype among patients who respond to 5-FU/oxaliplatin treatment $(22,23)$. In addition to drug response, several studies have demonstrated that MCRC patients with the GSTPI-105 A/G and G/G genotypes have favorable outcomes following oxaliplatin-based treatment compared with patients with the GSTP1-105 A/A genotype $(11,21)$. This tendency was also recognized in the results of the current study. The frequencies of the GSTP1-105 A/A, $\mathrm{A} / \mathrm{G}$ and $\mathrm{G} / \mathrm{G}$ polymorphisms were 70,25 and $3 \%$, respectively, in the Japanese population, which is similar to frequencies reported in other Asian populations, including Chinese and Taiwanese $(11,22,24)$. In American and European populations, there is an almost equal frequency of GSTPI-105 $\mathrm{A} / \mathrm{A}$ and $\mathrm{A} / \mathrm{G}$ carriers, which combine to make a total of $\sim 90 \%$ of all patients. By contrast, the frequency of the GSTP1-105 G/G genotype is $\sim 10 \%$ in these populations $(11-13,21,24)$. Regardless of ethnic differences, the association of the GSTP1-105 genotype with the clinical outcome is consistent among all MCRC patients who receive 5-FU/oxaliplatin as first-line chemotherapy.
In addition to GSTP1 and ERCC-1 and -2, members of the nucleotide excision repair pathway are involved in repair and tolerance of DNA damage and also encode key enzymes for oxaliplatin metabolism. Several studies have demonstrated that the ERCC1-118 and ERCC2-751 genotypes are associated with the clinical outcome of MCRC patients receiving oxaliplatin-based treatment $(11,13,18-20)$. In the present study, the ERCC2-751 genotypes were significantly associated with PFS, whereas no significant difference was identified between the ERCC1-118 genotype and the clinical outcome. The PFS of the patients with the ERCC2-751 A/A genotype was longer than that of patients with the ERCC2-751 A/C genotype, and this was consistent with previous studies $(11,13,20)$. The distribution of ERCC2-751 polymorphisms clearly differs between Asian and Western individuals. Among Asians, the frequencies of the ERCC2-751 A/A, A/C and C/C genotypes are 84-92, 8-16 and 0\%, respectively $(11,22)$, whereas among Americans and Europeans the frequencies are 25-38, 50-61 and 11-15\%, respectively $(11-13,20,22,23)$. There is a high possibility that the majority of Asians carry the ERCC2-751 A/A genotype, leading to promising outcomes of oxaliplatin-based chemotherapy.

A statistically significant association between $T S-3^{\prime}$-UTR genotypes and OS was identified in the current study. Treatment with FOLFIRI was also administered as second-line chemotherapy to $\sim 70 \%$ of patients receiving mFOLFOX6 treatment, and hence, the patients with increased survival rates were exposed to 5-FU for a long time. Numerous studies have indicated that MCRC patients with lower TS expression have a favorable outcome following 5-FU-based chemotherapy compared with patients with high $T S$ expression $(10,28)$. A previous study revealed that TS mRNA expression in rectal cancer patients with the TS-3'-UTR -6/-6 and -6/+6 genotypes was significantly lower compared with patients with the $T S-3$-UTR +6/+6 genotype, resulting in a favorable outcome following neoadjuvant 5-FU-based chemoradiation (29). Among CRC patients receiving 5-FU-based adjuvant treatment, the OS of patients with the TS-3'-UTR -6/-6 genotype was significantly longer compared with that of patients with other genotypes (30). Although there are various types of cancer, an encouraging association between clinical outcome and the TS-3'-UTR -6/-6 genotype has been identified in Asian gastric cancer patients receiving mFOLFOX6 treatment (31). Several studies have reported that there is no correlation between the TS-3'-UTR genotype and the clinical outcome of MCRC patients receiving 5-FU/oxaliplatin treatment (12-14,23). The frequency distribution of the TS-3'-UTR genotype may lead to discrepancies in the clinical outcome. In the current study, the frequencies of the TS-3'-UTR -6/-6, -6/+6 and +6/+6 genotypes were 38, 46 and $16 \%$, respectively, while in the USA and Europe these genotypes are $10-16,37-5$ and $33-53 \%$, respectively (11-13,23). Further studies may be required to clarify the association between these differences in ethnicity and the efficacy of anti-cancer drugs.

While mFOLFOX6 treatment improves the survival rate of MCRC patients, adverse events, including myelosuppression, nausea, diarrhea and peripheral neuropathy, are common. In particular, peripheral neuropathy, caused by cumulative administration of oxaliplatin, directly affects the quality of life and is a major reason for the discontinuation of oxaliplatin chemotherapy. Thus, predictive markers of peripheral neuropathy are required for prospective evaluations. In agreement 
with previous studies, the incidence of peripheral neuropathy higher than grade 2 was identified to significantly correlate with the GSTP1-105 and GSTM1 genotypes $(13,22)$. Notably, peripheral neuropathy in patients with the GSTPl-105 A/G and $\mathrm{G} / \mathrm{G}$ genotypes was of greater intensity compared with that of patients with the GSTPI-105 A/A genotype. A statistically significant correlation was identified between the GSTPI-105 genotype and the clinical outcome. Therefore, the GSTPI-105 polymorphism may serve as a double-edged marker for predicting response to 5-FU/oxaliplatin treatment and the intensity of oxaliplatin-associated peripheral neuropathy.

In the present study, the association among gene polymorphisms that affect the metabolism of 5-FU oxaliplatin and the clinical outcome in Japanese patients with MCRC was identified. Ethnic differences in the frequency distribution of polymorphisms, which preclude the extrapolation of clinical studies between Western and Asian populations, were also identified. Therefore, the present study is likely to improve chemotherapy for individuals of Asian descent. Consistent with studies in Western patients, the polymorphisms of GSTP1-105, ERCC2-751 and the 3'-UTR of TS were associated with the clinical outcome of FOLFOX treatment in Japanese MCRC patients. Therefore, these polymorphisms may be significant predictors of clinical outcome globally. However, GSTPI-105 and GSTM1 genotypes may be more useful as markers for severe oxaliplatin-induced peripheral neuropathy in Japanese patients compared with Western patients.

\section{References}

1. de Gramont A, Figer A, Seymour M, et al: Leucovorin and fluorouracil with or without oxaliplatin as first-line treatment in advanced colorectal cancer. J Clin Oncol 18: 2938-2947, 2000.

2. Tournigand C, André T, Achille E, et al: FOLFIRI followed by FOLFOX6 or the reverse sequence in advanced colorectal cancer: a randomized GERCOR study. J Clin Oncol 22: 229-237, 2004.

3. Goldberg RM, Sargent DJ, Morton RF, et al: A randomized controlled trial of fluorouracil plus leucovorin, irinotecan and oxaliplatin combinations in patients with previously untreated metastatic colorectal cancer. J Clin Oncol 22: 23-30, 2004

4. Reddy GK, Gibson AD and Price N: Evolution of FOLFOX regimens in the treatment of advanced colorectal cancer. Clin Colorectal Cancer 4: 296-299, 2005.

5. Shimizu T, Satoh T, Tamura K, et al: Oxaliplatin/fluorouracil/leucovorin (FOLFOX4 and modified FOLFOX6) in patients with refractory or advanced colorectal cancer: post-approval Japanese population experience. Int J Clin Oncol 2: 218-223, 2007.

6. Leichman CG, Lenz HJ, Leichman L, et al: Quantitation of intratumoral thymidylate synthase expression predicts for disseminated colorectal cancer response and resistance to protracted-infusion fluorouracil and weekly leucovorin. J Clin Oncol 15: 3223-3229, 1997.

7. Salonga D, Danenberg KD, Johnson M, et al: Colorectal tumors responding to 5-fluorouracil have low gene expression levels of dihydropyrimidine dehydrogenase, thymidylate synthase and thymidine phosphorylase. Clin Cancer Res 6: 1322-1327, 2000.

8. Shirota Y, Stoehlmacher J, Brabender J, et al: ERCC1 and thymidylate synthase mRNA levels predict survival for colorectal cancer patients receiving combination oxaliplatin and fluorouracil chemotherapy. J Clin Oncol 19: 4298-4304, 2001

9. Kim SH, Kwon HC, Oh SY, et al: Prognostic value of ERCC1, thymidylate synthase and glutathione S-transferase pi for 5-FU/oxaliplatin chemotherapy in advanced colorectal cancer. Am J Clin Oncol 32: 38-43, 2009.

10. Kumamoto K, Kuwabara K, Tajima Y, et al: Thymidylate synthase and thymidine phosphorylase mRNA expression in primary lesions using laser capture microdissection is useful for prediction of the efficacy of FOLFOX treatment in colorectal cancer patients with liver metastasis. Oncol Lett 3: 983-989, 2012.
11. Stoehlmacher J, Park DJ, Zhang W, et al: A multivariate analysis of genomic polymorphisms: prediction of clinical outcome to 5-FU/oxaliplatin combination chemotherapy in refractory colorectal cancer. Br J Cancer 91: 344-354, 2004.

12. Etienne-Grimaldi MC, Milano G, Maindrault-Goebel F, et al: Methylenetetrahydrofolate reductase (MTHFR) gene polymorphisms and FOLFOX response in colorectal cancer patients. Br J Clin Pharmacol 69: 58-66, 2010.

13. Ruzzo A, Graziano F, Loupakis F, et al: Pharmacogenetic profiling in patients with advanced colorectal cancer treated with first-line FOLFOX-4 chemotherapy. J Clin Oncol 25: 1247-1254, 2007.

14. Boige V, Mendiboure J, Pignon JP, et al: Pharmacogenetic assessment of toxicity and outcome in patients with metastatic colorectal cancer treated with LV5FU2, FOLFOX and FOLFIRI: FFCD 2000-05. J Clin Oncol 28: 2556-2564, 2010.

15. MaJ,StampferMJ,GiovannucciE, etal: Methylenetetrahydrofolate reductase polymorphism, dietary interactions and risk of colorectal cancer. Cancer Res 57: 1098-1102, 1997.

16. van der Put NM, Gabreëls F, Stevens EM, et al: A second common mutation in the methylenetetrahydrofolate reductase gene: an additional risk factor for neural-tube defects? Am J Hum Genet 62: 1044-1051, 1998.

17. Park DJ, Stoehlmacher J, Zhang W, et al: ERCC1 gene polymorphism is associated with differential ERCC1 gene expression. Proc AACR 93: 1591, 2002.

18. Park DJ, Zhang W, Stoehlmacher J, et al: ERCC1 gene polymorphism as a predictor for clinical outcome in advanced colorectal cancer patients treated with platinum-based chemotherapy. Clin Adv Hematol Oncol 1: 162-166, 2003.

19. Chang PM, Tzeng CH, Chen PM, et al: ERCCl codon $118 \mathrm{C}>\mathrm{T}$ polymorphism associated with ERCC1 expression and outcome of FOLFOX-4 treatment in Asian patients with metastatic colorectal carcinoma. Cancer Sci 100: 278-283, 2009.

20. Park DJ, Stoehlmacher J, Zhang W, et al: A Xeroderma pigmentosum group D gene polymorphism predicts clinical outcome to platinum-based chemotherapy in patients with advanced colorectal cancer. Cancer Res 61: 8654-8658, 2001.

21. Stoehlmacher J, Park DJ, Zhang W, et al: Association between glutathione S-transferase P1, T1 and M1 genetic polymorphism and survival of patients with metastatic colorectal cancer. J Natl Cancer Inst 94: 936-942, 2002.

22. Chen YC, Tzeng CH, Chen PM, et al: Influence of GSTP1 I105V polymorphism on cumulative neuropathy and outcome of FOLFOX-4 treatment in Asian patients with colorectal carcinoma. Cancer Sci 101: 530-535, 2010.

23. Zarate R, Rodríguez J, Bandres E, et al: Oxaliplatin, irinotecan and capecitabine as first-line therapy in metastatic colorectal cancer (mCRC): a dose-finding study and pharmacogenomic analysis. Br J Cancer 102: 987-994, 2010.

24. Watson MA, Stewart RK, Smith GB, et al: Human glutathione S-transferase P1 polymorphisms: relationship to lung tissue enzyme activity and population frequency distribution. Carcinogenesis 19: 275-280, 1998.

25. Eisenhauer EA, Therasse P, Bogaerts J, et al: New response evaluation criteria in solid tumours: revised RECIST guideline (version 1.1). Eur J Cancer 45: 228-247, 2009.

26. Moscow JA, Fairchild CR, Madden MJ, et al: Expression of anionic glutathione-S-transferase and P-glycoprotein genes in human tissues and tumors. Cancer Res 49: 1422-1428, 1989.

27. Ban N, Takahashi Y, Takayama T, et al: Transfection of glutathione S-transferase (GST)-pi antisense complementary DNA increases the sensitivity of a colon cancer cell line to adriamycin, cisplatin, melphalan and etoposide. Cancer Res 56: 3577-3582, 1996.

28. Popat S, Matakidou A and Houlston RS: Thymidylate synthase expression and prognosis in colorectal cancer: a systematic review and meta-analysis. J Clin Oncol 22: 529-536, 2004.

29. Stoehlmacher J, Goekkurt E, Mogck U, et al: Thymidylate synthase genotypes and tumour regression in stage II/III rectal cancer patients after neoadjuvant fluorouracil-based chemoradiation. Cancer Lett 272: 221-225, 2008.

30. Dotor E, Cuatrecases M, Martínez-Iniesta M, et al: Tumor thymidylate synthase 1494del6 genotype as a prognostic factor in colorectal cancer patients receiving fluorouracil-based adjuvant treatment. J Clin Oncol 24: 1603-1611, 2006.

31. Keam B, Im SA, Han SW, et al: Modified FOLFOX-6 chemotherapy in advanced gastric cancer: Results of phase II study and comprehensive analysis of polymorphisms as a predictive and prognostic marker. BMC Cancer 8: 148, 2008. 